\title{
Carotid sparing intensity modulated radiotherapy on early glottic cancer: preliminary study
}

\author{
Hoon Sik Choi, MD', Bae Kwon Jeong, MD ${ }^{1,2}$, Hojin Jeong, PhD ${ }^{1,2}$, Jin Ho Song, MD,2, \\ Jin Pyeong Kim, MD, PhD ${ }^{2,3}$, Jung Je Park, MD, PhD ${ }^{2,3}$, Seung Hoon Woo, MD, PhD ${ }^{2,3}$, Ki Mun Kang, MD, PhD 1,3 \\ 'Department of Radiation Oncology, Gyeongsang National University Hospital, Gyeongsang National University School of \\ Medicine, Jinju; ${ }^{2}$ Institute of Health Science, Gyeongsang National University, Jinju; ${ }^{3}$ Department of Otorhinolaryngology, \\ Gyeongsang National University Hospital, Gyeongsang National University School of Medicine, Jinju, Korea
}

Purpose: To compare the dose distribution between carotid sparing intensity modulated radiotherapy (IMRT) and opposed lateral field technique (LAFT), and to determine the effects of carotid sparing IMRT in early glottic cancer patients who have risk factors for atherosclerosis.

Materials and Methods: Ten early glottic cancer patients were treated with carotid sparing IMRT. For each patient, the conventional LAFT plan was developed for comparison. IMRT and LAFT plans were compared in terms of planning target volume (PTV) coverage, conformity index, homogeneity index, and the doses to planning organ at risk volume (PRV) for carotid arteries, spinal cord and pharyngeal constrictor muscle.

Results: Recurrence was not observed in any patients during the follow-up period. $V_{95 \%}$ for PTV showed no significant difference between IMRT and LAFT plans, while $\mathrm{V}_{100 \%}$ was significantly higher in the IMRT plan (95.5\% vs. 94.6\%, $\left.p=0.005\right)$. The homogeneity index (11.6\%) and conformity index (1.4) in the IMRT plan were significantly better than those in the LAFT plans (8.5\% and 5.1, respectively) $(p=0.005)$. The median $V_{56 y}(90.0 \%), V_{256 y}(13.5 \%)$, and $V_{506 y}(0 \%)$ for carotid artery PRV in the IMRT plan were significantly lower than those in the LAFT plan $(99.1 \%, 89.0 \%$, and $77.3 \%$, respectively) $(p=0.005)$.

Conclusion: Our study suggests that carotid sparing IMRT can significantly decrease the dose to carotid arteries compared to LAFT, and it would be considered for early glottic cancer patient with high risk of atherosclerosis.

Keywords: Carotid arteries, Intensity-modulated radiotherapy, Glottic cancer

\section{Introduction}

Glottic cancer is the most common malignant tumors of the larynx, with approximately 30\%-40\% tumors presenting in the early stage $[1,2]$. Currently, various treatment options have been used for management of early stage glottic cancer, including partial laryngectomy, transoral laser excision, and radiotherapy [1-3]. Among these methods, radiotherapy has been frequently chosen as the primary treatment because of its superiority in preserving the voice quality $[1,3]$. The conventional radiotherapy technique, opposed lateral field technique (LAFT), creates a uniform dose distribution on the beam pathways. This feature causes high radiation dose exposure in unnecessary areas. Thus, the risk of adverse effects can be increased in organs lying in the beam paths, such as carotid arteries, spinal cord, pharyngeal constrictor muscle or

Received 24 December 2015, Revised 5 February 2016, Accepted 3 March 2016.

Correspondence: Ki Mun Kang, MD, PhD, Department of Radiation Oncology, Gyeongsang National University Hospital, Gyeongsang National University School of Medicine, 79 Gangnam-ro, Jinju 52727, Korea. Tel: +82-55-750-9200, Fax: +82-55750-9095, E-mail: jsk92@gnu.ac.kr

(C) This is an Open Access article distributed under the terms of the Creative Commons Attribution Non-Commercial License (http://creativecommons.org/ licenses/by-nc/4.0/) which permits unrestricted non-commercial use, distribution, and reproduction in any medium, provided the original work is properly cited.

www.e-roj.org 
other organs at risk (OAR).

The radiation exposure during radiotherapy for early glottic cancer has been overlooked until recently because it often does not cause definite complications. However, because the rate of long-term complications caused by radiotherapy has increased with the increase in the proportion of long-term survivors and the quality of life has become more important among cancer survivors, the need for aggressive protection against treatment-related exposure during glottic cancer treatment has rapidly increased recently.

The potential risk of long term complications caused by radiation exposure of carotid arteries during head and neck cancer treatment has been reported in a previous study. Gujral et al. [4] reported that the thickness of carotid intima-media can be increased by radiation exposure, which causes an increase in carotid artery stenosis risk, thereby consequently increasing the risk of cerebrovascular accident. They suggested that risk for cerebrovascular injuries can be further increased in patients who have any risk factors for atherosclerosis.

In our study, in order to minimize the radiation dose to carotid arteries during treatment, we applied the intensity modulated radiotherapy (IMRT) technique, which helps to concentrate the radiation dose only to the tumor and to spare OAR. Here, we have presented the dosimetric characteristics and comparative results of the IMRT technique and the conventional LAFT, and have evaluated the feasibility of carotid sparing IMRT technique for treatment of early glottic cancer patients who have risk factors for atherosclerosis.

\section{Materials and Methods}

\section{Patients}

Ten early glottic cancer patients treated with carotid sparing IMRT at Gyeongsang National University Hospital between October 2013 and February 2015 were selected for the dosimetric analysis. Inclusion criteria for the study were that patients should have 1) histologically proven glottic cancer; 2) with T1No stage, on the basis of the 7th edition American Joint Committee on Cancer staging criteria; and 3) at least one of the following risk factors for atherosclerosis: hypertension, diabetes mellitus, hyperlipidemia, age over 70 years, smoking, or previous coronary heart disease. Patients with carcinoma higher stage than T2, Iymph node metastases, distant metastases, Eastern Cooperative Oncology Group performance score higher than 2, history of other malignant disease and previous history of radiotherapy for head and neck lesion were excluded. In all patients, physical examination, fiberoptic laryngoscopy, neck computed tomography (CT), tissue biopsy, neck magnetic resonance imaging and chest $\mathrm{CT}$ were routinely performed, and in some patients, 18-fluorodeoxyglucose positron emission tomography was performed.

\section{Radiotherapy}

All patients were immobilized in the supine position with a thermoplastic mask and CT images were scanned from the vertex to the sternoclavicular joint with 2-mm slice thickness. The CT images were then imported into the Eclipse treatment planning system ver. 10.0 (Varian Medical Systems, Palo Alto, CA, USA).

Target and OAR were contoured based on the guideline of University of Florida as summarized in Table 1 [5]. Clinical target volume (CTV) was contoured; superiorly, to include the most cranial extent of the arytenoid cartilage, inferiorly, to include from 1 to $1.5 \mathrm{~cm}$ below the level of the true vocal cords, posteriorly, to include the arytenoid cartilage and posterior commissure, and anteriorly and laterally, to include the anterior commissure while excluding the thyroid cartilage. A 3-mm margin was applied to CTV in all spherical axes, except in the posterior direction, to create the planning target volume (PTV). The carotid arteries and the spinal cord

Table 1. Delineation criteria for target volumes and OAR based on the University of Florida guidelines

\begin{tabular}{ll}
\hline Target & \multicolumn{1}{c}{ Border } \\
\hline CTV & Most cranial extent of the arytenoid cartilage \\
Superior & Extended from $1-1.5 \mathrm{~cm}$ below the level of the true vocal cord \\
Inferior & Encompassed the entirety of the arytenoid cartilage and posterior commissure \\
Posterior & Limited by the thyroid cartilage and included the anterior commissure \\
Lateral & CTV $+3 \mathrm{~mm}$ except for the posterior direction \\
PTV & Spinal cord and carotid arteries extended $1 \mathrm{~cm}$ superiorly and inferiorly beyond PTV \\
OAR & OAR volumes of carotid arteries were expanded by 3 mm uniformly \\
PRV &
\end{tabular}

OAR, organ at risk; CTV, clinical target volume; PTV, planning target volume; PRV, planning organ at risk volume. 

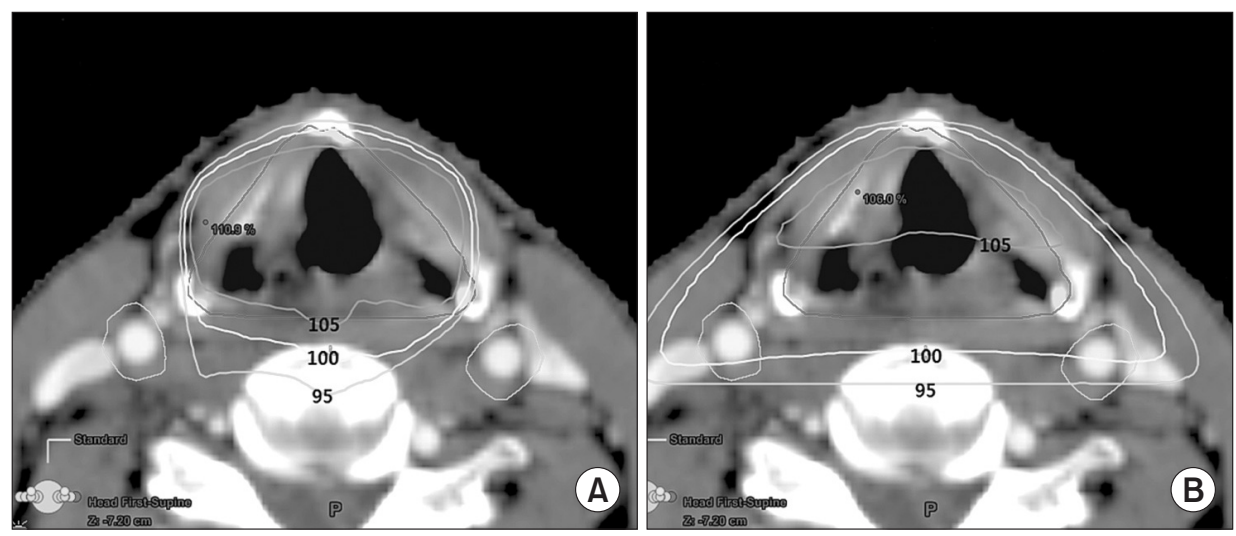

C

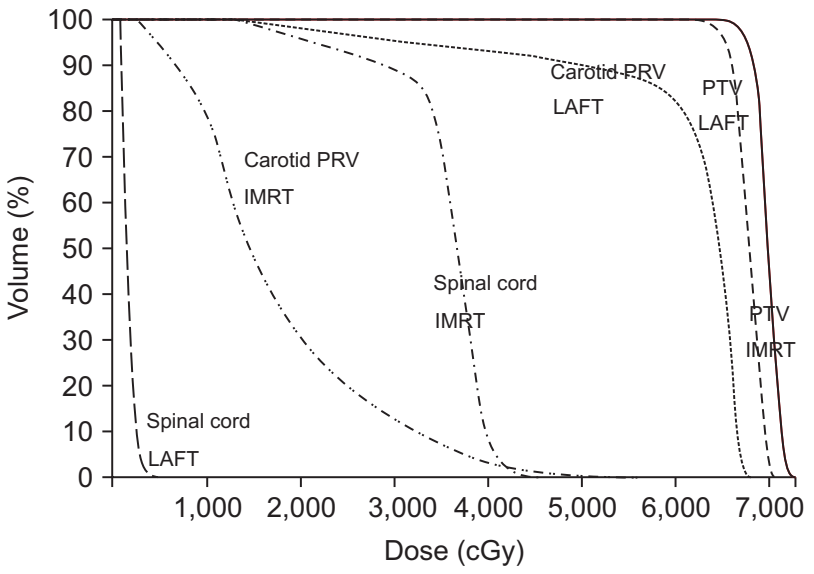

Fig. 1. Typical example of dose distributions in (A) IMRT, (B) LAFT plans, and (C) comparison of dose-volume histograms for the corresponding IMRT and LAFT plans. In (A) and (B), the region receiving at least 95\%, 100\%, and 105\% of the prescribed dose and contours for PTV (red lines), and PRVs of carotid arteries (green lines) are presented on an axial computed tomography slice of the patient. IMRT, intensity-modulated radiotherapy; LAFT, opposed lateral field technique; PTV, planning target volume; PRV, planning organ at risk volume.

were delineated as OARs. Both the carotid artery, spinal cord and pharyngeal constrictor muscle volumes were delineated up to $1-\mathrm{cm}$ superiorly and inferiorly beyond PTV. The carotid artery volumes were uniformly expanded by $3-\mathrm{mm}$ to create planning organ at risk volume (PRV). If the carotid artery PRV overlapped with PTV, PTV was manually modified to exclude the carotid artery PRV.

The IMRT plan was designed using 3 typical fields so that the PTV was adequately covered and the carotid artery PRV was spared as much as possible as shown in Fig. 1A. The anterior field was introduced to minimize the exposure of carotid arteries, and two oblique beams were tilted so that the ipsilateral carotid artery is out of field as much as possible. Six megavoltage X-ray energy and dynamic multileaf collimator were used. The carotid sparing IMRT plan was normalized so that $100 \%$ of the PTV received more than 95\% of the prescription dose. Table 2 shows the dose constraints; carotid
Table 2. Optimization criteria for intensity-modulated radiotherapy

\begin{tabular}{lcc}
\hline \multicolumn{1}{c}{ Target } & Dose (Gy) & Volume (\%) \\
\hline PTV max dose & 66.99 & 0 \\
PTV min dose & 62.7 & 100 \\
Carotid PRV dose & 30 & 20 \\
& 10 & 50 \\
Spinal cord max dose & 45 & 0 \\
\hline
\end{tabular}

PTV, planning target volume; PRV, planning organ at risk volume.

artery PRV was limited to $\mathrm{V}_{30 \mathrm{~Gy}}<20 \%$ and $\mathrm{V}_{10 \mathrm{~Gy}}<50 \%\left(\mathrm{~V} x_{\mathrm{Gy}}\right.$ : the percentage of the organ volume receiving $x$ Gy or more), and the spinal cord dose was limited to 45 Gy at any point All patients were equally prescribed a dose of 66 Gy in 33 fractions, 2 Gy per fraction. These patients were instructed not to swallow during radiotherapy to minimize organ motion, and the magnitude of the motion was confirmed by fluoroscopy with a radiographic simulator (Varian Medical Systems). For 
precise treatment, a megavoltage portal image using the electronic portal imaging devices was acquired at every week during the entire treatment course.

For the purpose of comparison, the conventional LAFT plan was created for each patient, as shown in Fig. 1B, while keeping the target volume, OAR and prescription dose the same as that in the carotid sparing IMRT. Two lateral opposed fields were used with wedges and were equally weighted in the LAFT plan. All LAFT plans were normalized so that the isocenter received $100 \%$ of the prescription dose.

\section{Dosimetric analysis}

IMRT and LAFT plans were compared relative to each other in terms of PTV coverage, conformity index, homogeneity index and doses to the carotid artery PRV, spinal cord and pharyngeal constrictor muscle. PTV coverage was analyzed using the terms $V_{95 \%}$, and $V_{100 \%}$ for PTV ( $V_{x_{0}}$ : the relative organ volume receiving $x$ percentage of the prescription dose). The conformity and homogeneity indices for all the plans were calculated using the following formula according to the definition of ICRU 83.

$$
\text { Conformity index }=\frac{\text { Treatment volume }}{\text { PTV }}
$$

where treatment volume is defined as the volume irradiated more than $95 \%$ of the prescription dose [5].

$$
\text { Homogeneity index }=\left(D_{2 \%}-\frac{D_{98 \%}}{D_{100 \%}}\right) \times 100 \%
$$

where $D_{2 \%}, D_{98 \%}, D_{100 \%}$ were defined as the minimum doses delivered to $2 \%$ and $98 \%$ of PTV, and prescription dose to PTV, respectively [6].

To quantify the carotid artery sparing, we calculated and compared the $\mathrm{V}_{5 \mathrm{~Gy}}, \mathrm{~V}_{25 \mathrm{G} y}, \mathrm{~V}_{50 \mathrm{~Gy}}$ and mean dose of carotid artery PRV. The spinal cord dose was compared based on the maximal spinal cord dose. The sparing of pharyngeal constrictor muscle was compared based on the mean dose and $V_{50 G y}$.

\section{Follow-up and statistical analysis}

During the course of radiotherapy, patients were evaluated weekly to assess acute toxicities. Patients were also followed up for 1, 2 and 3 months after completion of radiotherapy to assess radiation toxicities. Radiotherapy related toxicity was assessed according to the Common Terminology Criteria for Adverse Events (CTCAE) ver. 4.0 criteria [7]. After completion of radiotherapy, patients were followed up with clinical examination every 1 month and CT scan and magnetic resonance imaging were routinely performed every 3 to 6 months.

Comparisons between several dosimetric quantities for PTV and OAR of IMRT and LAFT were calculated by the Wilcoxon signed-rank test using SPSS software ver. 21.0 (SPSS Inc., Chicago, IL, USA) and p-value of less than 0.05 was considered statistically significant for all analyses.

\section{Results}

\section{Patient characteristics}

The characteristics of 10 patients are listed in Table 3. Patient median age was 62 years (range, 41 to 79 years) and all patients were males. All patients had more than one risk factor for atherosclerosis.

\section{Treatment outcomes}

The follow-up duration ranged between 4 and 17 months, and the median follow-up duration was 10 months. No recurrence was detected at the last follow-up for carotid sparing IMRT. No grade 3 or higher toxicity occurred during this study. Only grade 1 to 2 adverse skin reactions and mucositis occurred during radiotherapy; seven patients experienced grade 1 skin reactions, two patients experienced grade 2 skin reactions and grade 1 mucositis. All acute toxicities were successfully managed with supportive care. All patients received the planned radiotherapy dose without discontinuation or delay.

\section{Dosimetric comparison}

Fig. 1 shows an example of dose distributions and dose-volume histograms of IMRT and LAFT plans with the target and OAR contoured. The regions receiving at least 95\% of the prescribed

Table 3. Patient characteristics

\begin{tabular}{lccl}
\hline & Age (yr) & T stage & \multicolumn{2}{l}{ Risk factor for atherosclerosis } \\
\hline Patient 1 & 60 & 1a & Smoker \\
Patient 2 & 57 & 1b & DM, HTN, smoker \\
Patient 3 & 79 & 1a & DM, HTN, smoker \\
Patient 4 & 72 & 1a & HTN, smoker \\
Patient 5 & 67 & 1b & HTN, smoker \\
Patient 6 & 41 & 1b & DM, smoker \\
Patient 7 & 57 & 1a & Smoker \\
Patient 8 & 73 & 1a & Previous vascular disease, smoker \\
Patient 9 & 62 & 1b & Hyperlipidemia \\
Patient 10 & 55 & 1a & DM \\
\hline
\end{tabular}

DM, diabetes mellitus; HTN, hypertension. 
dose are denoted by a line.

1) PTV coverage: The dosimetric data for PTV coverage $\left(V_{95 \%}, V_{100 \%}\right)$, conformity index and homogeneity index are shown in Table 4. The median $V_{95 \%}$ values for IMRT and LAFT plans were 99.5\% (range, 99.1\% to 99.9\%) and 99.7\% (range, $99.2 \%$ to $99.9 \%$ ), respectively, with no significant difference $(p=0.209)$. However, the $V_{100 \%}$ values were higher in the IMRT plans (95.5\%; range, 95.3\% to $95.7 \%$ ) compared to the LAFT plans (94.6\%; range, 93.9\% to $94.7 \%$ ) with statistical significance $(p=0.005)$. The median conformity index was 1.4 (range, 1.1 to 1.9 ) in the IMRT plans compared to 5.1 (range, 4.7 to 5.5 ) in the LAFT plans ( $p=0.005$ ). The median homogeneity indices were $11.6 \%$ (range, $11.0 \%$ to $12.1 \%$ ) and $8.5 \%$ (range, $7.9 \%$ to $9.1 \%$ ) in the IMRT and LAFT plans, respectively ( $p=$ 0.005). All these differences were statistically significant.

2) Carotid artery PRV dose: The doses for the carotid artery PRV with the 2 different plans are shown in Table 4. The median carotid artery PRV dose was $14.7 \mathrm{~Gy}$ (range, 13.4 to $15.7 \mathrm{~Gy}$ ) for the IMRT plan and $53.9 \mathrm{~Gy}$ (range, 51.3 to $60.2 \mathrm{~Gy}$ ) for the LAFT plan; thus, it was significantly lower in the IMRT plan compared to the LAFT plan $(p=0.005)$. Carotid artery PRV $V_{5 G y^{\prime}} V_{25 G y^{\prime}}$ and $V_{50 G y}$ were $90.0 \%$ (range, 84.5\% to $94.8 \%$ ), 13.5\% (range, $8.1 \%$ to 15.3\%), and 0\% for the IMRT plan compared with $99.1 \%$ (range, $96.8 \%$ to $100 \%$ ), $89.0 \%$ (range, $85.1 \%$ to 95.5\%), and $77.3 \%$ (range, $50.1 \%$ to $83.4 \%$ ), respectively for the LAFT plan. There was a statistically significant reduction in volume of the carotid artery PRV irradiated when using IMRT than LAFT at all dose levels ( $p=0.005)$.

3) Spinal cord dose: In contrast to carotid arteries, the spinal cord dose was significantly higher in the IMRT plan. The maximum spinal cord dose was median $37.2 \mathrm{~Gy}$ (range, 35.7 to $39.1 \mathrm{~Gy}$ ) for the IMRT plans, and $2.5 \mathrm{~Gy}$ (range, 1.5 to $3.3 \mathrm{~Gy}$ ) for the LAFT plans. But, all values were below the spinal cord threshold dose of $45 \mathrm{~Gy}$.

4) Pharyngeal constrictor muscle: The median value of mean doses to pharyngeal constrictor muscle was 61.2 Gy (range, 55.9 to $66.1 \mathrm{~Gy}$ ) for the IMRT plans, and $64.6 \mathrm{~Gy}$ (range, 61.2 to $69.8 \mathrm{~Gy}$ ) for the LAFT plans. $\mathrm{V}_{50 \mathrm{~Gy}}$ of pharyngeal constrictor muscle was $88.7 \%$ (range, $77.3 \%$ to 93.7\%) for the IMRT plans, and 99.8\% (range, 90.3\% to 99.9\%) for the LAFT plans. These two values were significantly higher in the LAFT plan $(p=0.009$ and $p=0.007)$.

\section{Discussion and Conclusion}

Carotid arteries lying in the beam pathway of conventional radiotherapy for early glottic cancer are exposed to relatively high doses almost identical to the target dose. There are several reports showing the relation between radiotherapy and carotid artery stenosis. Brown et al. [8] reported that

Table 4. Dosimetric data for target volume and OAR in IMRT and LAFT plans

\begin{tabular}{lccc}
\hline & IMRT plan & LAFT plan & p-value \\
\hline PTV (\%) & & & \\
$V_{95 \%}$ & $99.5(99.1-99.9)$ & $99.7(99.2-99.9)$ & 0.209 \\
$V_{100 \%}$ & $95.5(95.3-95.7)$ & $94.6(93.9-94.7)$ & 0.005 \\
$\mathrm{HI}(\%)$ & $11.6(11.0-12.1)$ & $8.5(7.9-9.1)$ & 0.005 \\
Cl & $1.4(1.1-1.9)$ & $5.1(4.7-5.5)$ & 0.005 \\
Mean Carotid PRV dose (Gy) & $14.7(13.4-15.7)$ & $53.9(51.3-60.2)$ & 0.005 \\
Carotid PRV (\%) & & \\
V $_{56 y}$ & $90.0(84.5-94.8)$ & $99.1(96.8-100)$ & 0.005 \\
V $_{256 y}$ & $13.5(8.1-15.3)$ & $89.0(85.1-95.5)$ & 0.005 \\
$V_{506 y}$ & $0(0-0)$ & $77.3(50.1-83.4)$ & 0.005 \\
Maximum spinal cord dose (Gy) & $37.2(35.7-39.1)$ & $2.5(1.5-3.3)$ & 0.005 \\
Mean PCM dose (Gy) & $61.2(55.9-66.1)$ & $64.6(61.2-69.8)$ & 0.009 \\
PCM (\%), $V_{50 G y}$ & $88.7(77.3-93.7)$ & $99.8(90.3-99.9)$ & 0.007 \\
\hline
\end{tabular}

Values are presented as median (range).

$V_{x \%}$ : the relative organ volume receiving $x$ percentage of the prescription dose.

$V_{x G y}$ : the percentage of the organ volume receiving $x$ Gy or more.

OAR, organ at risk; IMRT, intensity modulated radiotherapy; LAFT, opposed lateral field technique; PTV, planning target volume; HI, homogeneity index; $\mathrm{Cl}$, conformity index; PRV, planning organ at risk volume; PCM, pharyngeal constrictor muscle. 
the incidence of carotid artery stenosis was higher in the irradiated neck than that in the contralateral unirradiated neck. Cheng et al. [9] reported that the risk of developing carotid artery stenosis was increased in patients who undergo irradiation of the neck for more than 5 years. In addition, some reports showed that radiotherapy increased the risk of cerebrovascular accident. Smith et al. [10] reported that the 10-year incidence of cerebrovascular events was increased by 9\% in head and neck cancer patients when they were treated with radiotherapy. Dorresteijn et al. [11] also reported that the 15-year cumulative risk of stroke after radiotherapy to the neck was 12\% and there was a 5.6 times higher chance of stroke in patients who had undergone neck radiotherapy than those who had not undergone neck radiotherapy. Cheung [12] reported that the risk factors for frequent carotid artery stenosis after radiotherapy included old age, smoking, coronary heart disease, and previous stroke.

Hence, in order to reduce the carotid artery dose for early glottic cancer treatment, some studies have adopted the carotid sparing techniques by using modified oblique field, IMRT, arc therapy or tomotherapy instead of conventional radiotherapy (Table 5) [13-15]. Rosenthal et al. [16] first reported their carotid sparing IMRT for early glottic cancer. They treated 11 patients with T1-2 glottic cancer, and suggested that IMRT significantly reduced radiation dose to the carotid arteries compared to conventional techniques. They concluded that IMRT techniques are necessary, especially for younger patients. Another study on carotid sparing was published by Chera et al. [17] from the University of Florida. They compared the dosimetric results between opposed lateral fields, three-dimensional conformal radiotherapy, and IMRT plan. They also suggested that IMRT can reduce the dose to carotid arteries. Recently, Zumsteg et al. [18] reported the dosimetric as well as the clinical results of 48 patients who were treated with carotid sparing IMRT. They found that the 3 -year local control rate was $88 \%$, which was similar to that for conventional radiotherapy. They suggested that carotid sparing IMRT was a safe and effective treatment method.

Our study also shows that the carotid artery dose was much lower with carotid sparing IMRT than with LAFT for early glottic cancer. The exact tolerance dose and dose-volume relationship for carotid artery damage from radiation have not yet been established. However, in our study, the difference in the mean dose to carotid arteries between IMRT and LAFT was very large, approximately $50 \mathrm{~Gy}$ and the dose in the IMRT plan was lower at all dose levels. Therefore, we suggest that carotid sparing IMRT could reduce the risk of carotid artery damage

http://dx.doi.org/10.3857/roj.2016.34.1.26

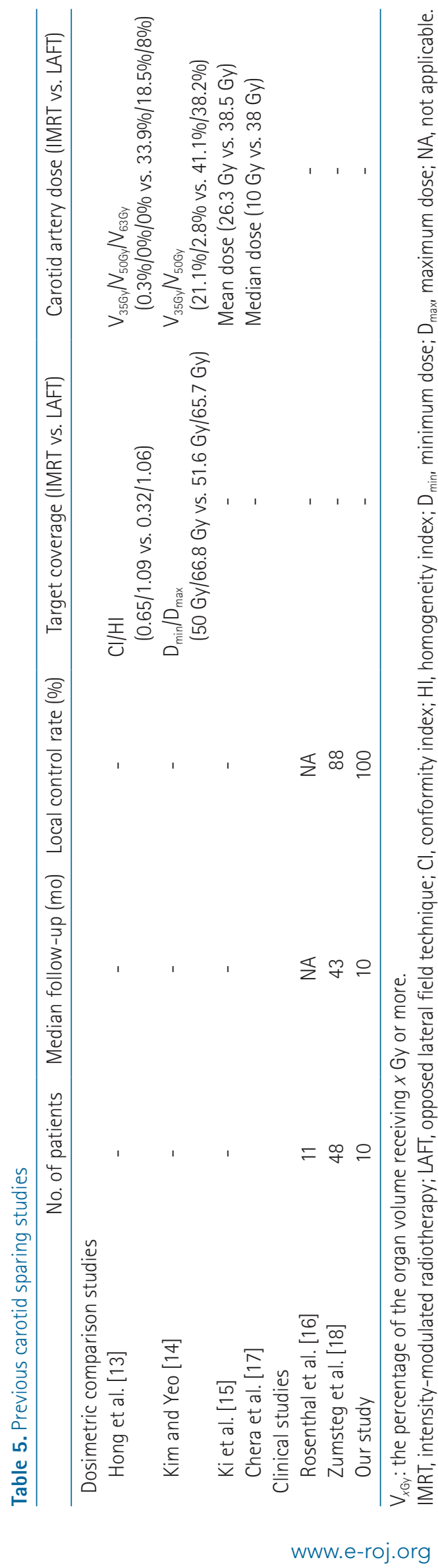


and stenosis. In addition, since our patients had at least one risk factor for atherosclerosis, we can expect reduction in the incidence of cerebrovascular accidents. We found that all treatment plans had satisfactory PTV coverage, but carotid sparing IMRT plans had significantly high $\mathrm{V}_{100 \%}$ values for PTV. Also, the carotid sparing IMRT plan achieved good conformity and favorable homogeneity for PTV coverage than the LAFT plan. For the IMRT plan, spinal cord dose was significantly higher than that for the LAFT plan, but all values were below the threshold dose of $45 \mathrm{~Gy}$. Mean dose and $\mathrm{V}_{50 \mathrm{~Gy}}$ values to the pharyngeal constrictor muscle were significantly higher in the LAFT plan. No recurrent as well as no severe toxicities including dysphagia were observed in these 10 early glottic cancer patients with good compliance. On the basis of these data, we expect that carotid sparing IMRT could reduce carotid artery damage in the long-term follow-up while achieving acceptable local control, toxicity, and compliance.

However, there are some concerns about IMRT for treatment of early glottic cancer. The first concern is the risk of underdosage because of intrafraction movement. Several studies have reported about the intrafraction movement of the larynx during swallowing [16,19-21]. This motion is more important with IMRT due to longer treatment times and high conformable target coverage. Therefore, several carotid sparing studies reported about the concern of intrafraction motion from mobile larynx during IMRT $[17,18]$. We trained the patients to hold swallowing during the entire course of treatment. And, also examined the dynamic organ motion through the radiographic simulator. This education on swallowing may be helpful in reducing the risk of target motion. In addition, for minimizing the intrafraction movement, we reduce the treatment time through a relatively simple carotid sparing IMRT plan with 3 fields. Otherwise, in the aspect of interfraction set-up error, we used 3-mm PTV expansions margins with weekly portal films. Some studies reported that $5-\mathrm{mm}$ margin is needed to compensate the setup error if the larynx is a target, and usually verified the larynx motion by daily image such as portal films or in room CT $[14,18,22]$. However, by analyzing the log files of the couch movement after the acquisition of weekly portal films, we observed that weekly couch variation was within $3 \mathrm{~mm}$ (mean, $2.3 \pm 0.8$ ).

The second concern is the question of clinical effectiveness. The adverse effects of radiation on carotid arteries can be observed over several years, Teshima et al. $[3,23,24]$ and Osman et al. [19] reported that vascular effects can manifest more than 10 years after radiotherapy. This late vascular damage was induced not only by radiotherapy but also by several cofactors such as smoking and cholesterol. Hence, adverse effects of radiation on carotid arteries were confusing. Surveillance with vascular imaging such as ultrasound examinations and CT are needed to detect carotid artery damage, but periodic examination included baseline study was difficult. Thus, evaluation of the exact clinical effectiveness of carotid sparing IMRT is very difficult. Therefore, there is still no definitive data showing the relation between carotid artery dose and vascular effects, and the clinical impact of carotid sparing IMRT. To prove the clinical effectiveness of carotid sparing IMRT, we need to perform a study with more number of patients, proper methods of examination of the carotid artery damage, and long-term follow-up of more than 10 years.

In conclusion, this preliminary study shows that carotid sparing IMRT achieves a satisfactory disease control rate and significantly reduces the radiation dose to carotid arteries to a large extent. We suggest that carotid sparing IMRT in early glottic cancer is feasible, and it can be a more reasonable choice for patients who have risk factors for atherosclerosis.

\section{Conflict of Interest}

No potential conflict of interest relevant to this article was reported.

\section{References}

1. Mendenhall WM, Mancuso AA, Amdur RJ, Werning JW. Laryngeal cancer. In: Halperin EC, Brady LW, Perez CA, et al., editors. Perez and Brady's principles and practice of radiation oncology. 6th ed. Philadelphia, PA: Lippincott Williams \& Wilkins; 2013. p. 850-68.

2. Petrakos I, Kontzoglou K, Nikolopoulos TP, Papadopoulos 0 , Kostakis A. Glottic and supraglottic laryngeal cancer: epidemiology, treatment patterns and survival in 164 patients. J BUON 2012;17:700-5.

3. Teshima T, Chatani M, Hata K, Inoue T. Radiation therapy of early glottic cancer (T1NOMO); retrospective review of historical control. Rinsho Hoshasen 1989;34:1603-6.

4. Gujral DM, Shah BN, Chahal NS, Senior R, Harrington KJ, Nutting CM. Clinical features of radiation-induced carotid atherosclerosis. Clin Oncol (R Coll Radiol) 2014;26:94-102.

5. Chatterjee S, Guha S, Prasath S, Mallick I, Achari R. Carotid sparing hypofractionated tomotherapy in early glottic cancers: refining image guided IMRT to improve morbidity. J Cancer Res Ther 2013;9:452-5.

6. Lauve A, Morris M, Schmidt-Ullrich R, et al. Simultaneous integrated boost intensity-modulated radiotherapy for locally advanced head-and-neck squamous cell carcinomas: II. 
clinical results. Int J Radiat Oncol Biol Phys 2004;60:374-87.

7. Chen AP, Setser A, Anadkat MJ, et al. Grading dermatologic adverse events of cancer treatments: the Common Terminology Criteria for Adverse Events Version 4.0. J Am Acad Dermatol 2012;67:1025-39.

8. Brown PD, Foote RL, McLaughlin MP, et al. A historical prospective cohort study of carotid artery stenosis after radiotherapy for head and neck malignancies. Int J Radiat Oncol Biol Phys 2005;63:1361-7.

9. Cheng SW, Ting AC, Lam LK, Wei WI. Carotid stenosis after radiotherapy for nasopharyngeal carcinoma. Arch Otolaryngol Head Neck Surg 2000;126:517-21.

10. Smith GL, Smith BD, Buchholz TA, et al. Cerebrovascular disease risk in older head and neck cancer patients after radiotherapy. J Clin Oncol 2008;26:5119-25.

11. Dorresteijn LD, Kappelle AC, Boogerd W, et al. Increased risk of ischemic stroke after radiotherapy on the neck in patients younger than 60 years. J Clin Oncol 2002;20:282-8.

12. Cheung K. Intensity modulated radiotherapy: advantages, limitations and future developments. Biomed Imaging Interv J 2006;2:e19.

13. Hong CS, Oh D, Ju SG, et al. Carotid-sparing tomohelical 3-dimensional conformal radiotherapy for early glottic cancer. Cancer Res Treat 2016;48:63-70.

14. Kim ES, Yeo SG. Volumetric modulated arc radiotherapy sparing the thyroid gland for early-stage glottic cancer: a dosimetrical analysis. Oncol Lett 2014;7:1987-91.

15. Ki YK, Kim WT, Nam JH, et al. Treatment planning for minimizing carotid artery dose in the radiotherapy of early glottic cancer. J Korean Soc Ther Radiol Oncol 2011;29:115-20.

16. Rosenthal DI, Fuller CD, Barker JL Jr, et al. Simple carotid-sparing intensity-modulated radiotherapy technique and preliminary experience for T1-2 glottic cancer. Int J Radiat Oncol Biol Phys
2010;77:455-61.

17. Chera BS, Amdur RJ, Morris CG, Mendenhall WM. Carotidsparing intensity-modulated radiotherapy for early-stage squamous cell carcinoma of the true vocal cord. Int J Radiat Oncol Biol Phys 2010;77:1380-5.

18. Zumsteg ZS, Riaz N, Jaffery S, et al. Carotid sparing intensitymodulated radiation therapy achieves comparable locoregional control to conventional radiotherapy in T1-2No laryngeal carcinoma. Oral Oncol 2015;51:716-23.

19. Osman SO, Astreinidou E, de Boer HC, et al. IMRT for imageguided single vocal cord irradiation. Int J Radiat Oncol Biol Phys 2012;82:989-97.

20. van Asselen $B$, Raaijmakers $C P$, Lagendijk JJ, Terhaard CH. Intrafraction motions of the larynx during radiotherapy. Int J Radiat Oncol Biol Phys 2003;56:384-90.

21. Kwa SL, Al-Mamgani A, Osman SO, Gangsaas A, Levendag PC, Heijmen $B J$. Inter- and intrafraction target motion in highly focused single vocal cord irradiation of t1a larynx cancer patients. Int J Radiat Oncol Biol Phys 2015;93:190-5.

22. Baron CA, Awan MJ, Mohamed AS, et al. Estimation of daily interfractional larynx residual setup error after isocentric alignment for head and neck radiotherapy: quality assurance implications for target volume and organs-at-risk margination using daily CT on- rails imaging. J Appl Clin Med Phys 2014;16:5108.

23. Teshima T, Chatani M, Inoue T. Radiation therapy for early glottic cancer (T1NOMO): I. results of conventional open field technique. Int J Radiat Oncol Biol Phys 1989;17:1199-202.

24. Teshima T, Chatani M, Inoue T. Radiation therapy for early glottic cancer (T1NOM0): II. prospective randomized study concerning radiation field. Int J Radiat Oncol Biol Phys 1990; 18:119-23. 